\title{
The "Danger" of Consensus Messaging: Or, Why to Shift From Skeptic-First to Migration-First Approaches
}

\author{
Chris Russill* \\ Communication and Media Studies, School of Journalism and Communication, Carleton University, Ottawa, ON, Canada
}

\section{OPEN ACCESS}

Edited by:

Anabela Carvalho, University of Minho, Portugal

Reviewed by:

Pieter Maeseele, University of Antwerp, Belgium Peter Berglez, Jönköping University, Sweden

*Correspondence: Chris Russill chris.russill@carleton.ca

Specialty section: This article was submitted to Science and Environmental

Communication,

a section of the journal Frontiers in Communication

Received: 10 June 2017 Accepted: 06 August 2018 Published: 24 August 2018

Citation:

Russill C (2018) The "Danger" of Consensus Messaging: Or, Why to

Shift From Skeptic-First to

Migration-First Approaches.

Front. Commun. 3:37.

doi: 10.3389/fcomm.2018.00037
Consensus messaging is a climate change communication strategy emphasizing the fact of scientific consensus on anthropogenic global warming (AGW). Its proponents encourage scientists, journalists, and educators to transmit consensus messages in hopes of improving public climate literacy. Critics of this approach question its methodology for determining consensus and its effectiveness as a strategy for improving public understanding and policymaking. I review these debates to determine what is at stake in disagreements over consensus messaging and suggest that issues of climate change danger are addressed too narrowly when the expectations, style and categories of consensus messaging are dominant. I recommend that "migration-first" approaches displace the priority of "skeptic-first" approaches to climate change communication, and that scholars begin asking what is owed to those most affected by climate change danger.

Keywords: climate change communication, cultural cognition, environmental humanities, risk communication, climate change refugees, climate change policy, environmental politics, migration

Climate change communication is an intensely contested terrain. As a growing object of scholarly interest, proposals to reshape its usual practices have proliferated rapidly and sharpened differences among experts regarding the nature and purposes of public communication on climate change. These disputes reflect differences in how communication is conceptualized among the disciplines (psychology, sociological, political science, cognitive science, communication, and media studies, etc.), how it works in various sites (science communication, intergovernmental negotiating, social marketing, social movements, risk communication, local politics, etc.) and what it is expected to do (combat skeptics, support mitigation polices, explore adaptation opportunities). Arguably, these disputes are symptomatic of a growing dissatisfaction with the conceptual narrowness of climate change communication given the expanding range of contexts, circumstances, and concerns it must address. In some cases, scholars are calling for agonistic approaches to reshape politics and public life through climate change communication (Carvalho and Peterson, 2012; Machin, 2013).

In this article, I address scholarly debates about "consensus messaging" to discuss current limitations in climate change communication. While these debates often turn on how the purposes and goals of climate change communication are understood, they illustrate the narrow origins, limited intentions and general ineffectiveness of the field. Alternative approaches, I suggest, should reckon directly with the legacy and deeper implications of consensus messaging for climate change communication, not lament its popularity, dismiss its assumptions or ignore its influence. 
Of special importance is a reflexive awareness of how the expectations, style and contents of consensus messaging limit our ability to communicate danger. By encouraging a geophysical understanding of climate change to shape the opinions and participation of valued citizens, global temperature targets gained political support and now define dangerous anthropogenic inference with the climate system. Climate change danger requires a much broader approach and should shift our communicative priorities from shaping citizen opinion to addressing what is owed to those most affected. In this respect, climate change communication might encompass migration, forced dislocation and refuge as central problems mediating our relationship to climate change danger.

\section{CONSENSUS MESSAGING: APPROACH AND STRATEGY}

\author{
"Ninety-seven percent of scientists agree: climate change is real, \\ man-made, and dangerous" Obama (2013)
}

Cook et al. (2013) promote a "consensus gap" approach to climate change communication, a strategy that quantifies the scientific consensus on anthropogenic global warming (AGW) at 97.2\%. Their messaging gained wide attention-even a "97\%" presidential tweet from Barack Obama (see above) - and has generated an interesting set of debates.

The strategy is simple. Cook et al. (2013) believe the scientific consensus on AGW is grossly underestimated by journalists, educators and the public more generally. They quantify expert opinion to illustrate a "consensus gap" in public misunderstanding and to call for its closure. Political inaction on climate change, on their approach, is rooted in decades of distorted communication, a diagnosis calling for climate literacy. Cook and his colleagues address one effect of this distortion without dwelling at length on its origins, causes or consequences. If we are disciplined in our efforts to reduce the consensus gap, and if we embolden scientists, educators and journalists to discuss climate change in this confident and straightforward way, an informed public opinion will encourage politicians to pursue effective policy change. It is an approach popular in the United States and designed to intervene in its political context.

The strategy is a novel twist on an older set of assumptions. Consensus messaging is encouraged by the United Nations Intergovernmental Panel on Climate Change (IPCC), which produced a scientific consensus on AGW through deliberative means in 1995 (and in 2001, and again in 2007 and so on). These reports were met by skeptical challenges almost immediately. Alarmed by the persistence of contrarian claims after the third IPCC assessment in 2001, Oreskes (2004) addressed the issue in a forthright way. She sampled abstracts of the primary science along with official statements from scientific organizations. She found no opposition to AGW and conveyed the results in a single page of prose using an easily reproducible methodology.

Oreskes (2004) paper should have closed the question. Instead, it is the origin story for an expanding sub-field of climate change communication. There are perhaps ten methodologies for assessing the scientific consensus on AGW, and scholarly studies of consensus messaging as a communication strategy have emerged, grown in sophistication, and become a field of persistent debate in blogs, professional meetings and the primary research literature.

Cook et al. (2013) are notable in this context for systemizing consensus messaging around a quantitative measure, for shaping subsequent research and advocacy efforts, and for gaining wide attention. Current debate takes their study as a point of departure in refining methodological questions (Cook et al., 2014; Skuce et al., 2016), optimizing consensus messaging as a communication strategy (van der Linden et al., 2014), and seeking empirical evidence of its effectiveness in shifting public opinion (Ding et al., 2011; McCright et al., 2013; Maibach et al., 2014; van der Linden et al., 2015; Cook and Lewandowsky, 2016; Deryugina and Shurchkov, 2016).

\section{CONSENSUS MESSAGING: CRITIQUE AND ALTERNATIVES}

The popularity of the "consensus gap" strategy has drawn the attention of critics and provoked debate. Critical questions were raised initially in the back and forth of blogs, conference talks, and gray literature, but have spilled more recently into congressional hearings (in the U.S.) and the scholarly literature. While the consensus approach is emblematic of what is sometimes called an instrumental or deficit model approach to communication (Carvalho and Peterson, 2012; Machin, 2013; Maeseele, 2013, 2015), I reflect here on specific disputes over its methodological and strategic orientation.

\section{Methodology}

Different methodologies for quantifying the degree of scientific consensus produce different results. These differences encourage critics to question the rigor with which Cook et al. (2013) carried out their study, most notably Tol (2014, 2016). In brief, Tol suggests the enumerated consensus is too high and an artifact of methodology, not an accurate reflection of the scientific community. James (Powell, 2015) uses similar reasoning to draw the opposite conclusion. Using a different method, Powell claims 99.99\% of publishing scientists agree and that there is virtual unanimity on AGW.

The dispute is useful for demonstrating how different perspectives on the efficacy of climate change communication are fought out on methodological terrain. In Powell's view, the idea that nearly $3 \%$ of relevant scientists might question $A G W$ is intolerable. It invites skepticism that "could weaken the case for action to prevent global warming" (p. 121). Given that unanimous science is rarely if ever overturned (Powell, 2016), it would be wiser to convince citizens of the unanimity among scientists lest skeptics retain a foothold in public discourse. Tol's efforts would be one example.

Cook et al. (2016) respond by emphasizing the gap between scientific and public understanding, not the percentage of scientists unconvinced by the scientific consensus. While the difference between 97 and $99.99 \%$ is inconsequential from this perspective, Cook and his collaborators do criticize Powell 
for minimizing the existence of contrarian scientists as it "exaggerates the true level of scientific consensus on AGW" (Skuce et al., 2016, p. 5). Powell's method, they conclude, "leaves the study of consensus vulnerable to criticism and could lead to further public confusion," as quibbling over the degree of consensus distracts from the fact of consensus, however quantified (p. 5).

The dispute turns on the anticipated effects of their different measures (97 or $99.99 \%$ ) to climate change communication, not a desire for precision in measuring scientific consensus. Their primary concern is confronting skepticism and denial in its implications for public discourse on climate change. By systematizing climate change communication around displays of consensus or unanimity, the strategy hopes to ward of what is feared (the influence of climate skepticism) rather than address those most affected by the consequences of climate change.

\section{Strategy}

While disputes over methodology are often proxy conflicts for differing approaches to climate change communication, scholars have raised more explicit concerns with the strategy. It is not clear that disputes resulting from political inaction on climate change are resolvable by claims to scientific consensus; in fact, for many critics, this strategy is not simply a mistake but makes public communication of climate change significantly more difficult (Machin, 2013; Howe, 2014; Maeseele, 2015; Russill, 2016; Heymann et al., 2017; Pearce et al., 2017). I focus here on the criticism of Dan Kahan and Mike Hulme not to slight other approaches but to address the most vocal critics of consensus messaging.

Kahan (2015) emphasizes the importance of cultural values and identity to climate change communication from a social science perspective. People are easy to polarize politically on questions of climate change because they are animated by different cultural values. Messages prioritizing the scientific consensus on climate change do not transcend political polarization by replacing ideological commitments with public literacy; these efforts intensify conflict by reproducing the very communicative dynamics that polarize public discourse on climate change.

On this account, then, what most scientists believe is simply another empirical fact no different from any other that bears on a disputed question of risk. As such, scientific consensus cannot be expected to counteract the polarizing effects of cultural cognition because apprehension of it will necessarily occur through the same social psychological mechanisms that shape individuals' perceptions of every other manner of fact (Kahan et al., 2011, p. 4).

Consensus messaging, in this respect, fails to achieve its goals and perpetuates a communicative relationship where no one else can succeed either. On Kahan's approach, communication should first contribute to the maintenance of cultural contexts that affirm our identities and values as meaningful when seeking to discuss climate change. In his view, consensus messaging is unsupported by contemporary social science and ineffective in helping citizens engage with climate change.

Hulme (2015) is similarly concerned with "consensus entrepreneurs" misleading public discourse on climate change.
If Kahan criticizes consensus messaging as out of step with the study of risk communication, Hulme challenges its broader understanding of science and public life from a historicallyinformed science, technology and society (STS) perspective. The geophysical sciences defining the expert consensus on AGW are implicated in a cultural history that shapes both scientific and public concern with climate change. Weart (2003) charted key elements of this history in recounting the relationship of geophysics and environmentalism to the discovery of AGW. Hulme (2009) work develops a wider frame for the relationship and situates the communication of climate change within more agonistic conceptions of public life.

It is from this perspective that Hulme (2013) caricatures the dominant approach to climate change policy as "the plan," as Sarewitz (2011) had done previously. "The plan" assumes that public demonstrations of scientific consensus generate the political support necessary for effective climate policy. The role of public communication is to elevate the role of science and generate assent via symbolic displays of consensus. Just as Kahan finds this approach inconsistent with risk communication, Hulme suggests this strategy for policymaking is contradicted by research demonstrating how science circulates within contemporary democratic culture. Time and again, the reliance on scientific consensus to prescribe political action generates intense opposition in the form of skepticism toward knowledge (Howe, 2014). The result isn't an effect of public ignorance (as consensus messaging would presuppose); it is evidence that public life in contemporary democratic societies is fundamentally agonistic. The tragedy of consensus messaging is not an inability to overcome the agonism of politics; it is that political contention is mediated primarily by the categories, discourses, and institutions of the geophysical sciences, rather than by political and ethical discourse (see also Carvalho and Peterson, 2012; Howe, 2014; Maeseele, 2015).

Hulme's critique turns on an important question. Should communication generate assent to expert consensus or accept disagreement, difference and dispute as irreducible aspects of democratic culture? If agonistic conceptions of public life are valued, then experts, policymakers and citizens should accept diversity and dissent as intractable elements of communication. On this perspective, when we communicate climate change, we are not conveying information or expertise primarily, but working through political, cultural, and ethical differences. Communicative strategies should respect rather than suppress or dampen the significance of these differences.

Hulme's approach is a pluralistic variant on liberal democracy that treats disagreement and difference not as obstacles to be reduced, eliminated or ignored through persuasive communication, but as constitutive features of public life. To be sure, there are more radical variants of agonistic politics that would ground Hulme's language of disagreement and difference in theories of ideology, radical social movements and political economy (Carvalho and Peterson, 2012; Machin, 2013; Berglez and Olausson, 2014; Maeseele, 2015). In this work, the challenges posed by capitalist exploitation are more overt than in Hulme's approach. There is broad agreement however that climate change communication is embedded in systems of power that are deeply 
contested; disputes over the legitimacy of its strategies reflect differences in perspective on democracy and power.

\section{The "Danger" of Consensus Messaging}

In the work discussed above, climate change communication appears variously as (1) a matter of improving public literacy, (2) a vehicle of risk communication and environmental policymaking, and (3) a problem of coping with the intractable disagreement and antagonism of democratic politics. Opinions on the value of consensus messaging often depend on how we understand the purpose and goals of climate change communication.

The broader problem is the way consensus messaging creates expectations, styles and symbols of discourse that constrain our awareness of how communication is reshaped by the new political challenges that climate change is generating or amplifying (Carvalho and Peterson, 2012). Consensus messaging gains the attention of political elites and may improve survey measures of climate literacy, yet it is insufficient for the multiple and expanding contexts to which climate change communication is now answerable. If our work shifts from anxiety about skeptics to what is owed those most affected by climate change danger, the limitations of consensus messaging become still starker.

The question of danger is useful in assessing the legacy and deeper consequences of consensus messaging. It is a crucial element of climate change, as concerns with dangerous anthropogenic interference are encoded in the Framework Convention on Climate Change, yet its conceptualization is severely constrained by consensus messaging and other "science-first" approaches (Howe, 2014). I address this issue by considering the expectations, style and symbols of consensus messaging as they shape discourse on climate change danger before asking if a redirection of climate change communication is possible.

\section{Expectations of Consensus}

We can raise the problem of expectations by returning to Obama's tweet, the high point of public success for this strategy (Obama, 2013). The tweet recirculates the 97\% meme generated by Cook's methodology, yet Obama runs together the scientific consensus on AGW with the dangerousness of climate change. We might call this a mistake (there is no $97 \%$ consensus on climate change danger) or an opinion (it is a reasonable if disputable inference), yet it is symptomatic of deeper problems with consensus messaging.

Consider the following questions: if Obama had wished to tweet the consensus on climate change danger, what could he say? Should he enumerate the scientists agreeing with the twodegree threshold for the dangerousness of climate change? If the methods of determining consensus for AGW fail to produce a consensus on danger, is this a problem?

I pose these questions as the methods for determining the consensus on AGW are debated, refined and gain in scope and sophistication. It seems reasonable that these techniques would prove more authoritative as consensus messaging develops its methodology, shapes communicative strategy and steers public discourse.

\section{Style of Consensus}

The question of whether to enumerate consensus for issues of danger is a minor one. A larger problem is that the conditions of effectiveness for consensus messaging create expectations and norms for communicative style that are unworkable for questions of danger. Consensus messaging prioritizes indisputable facts that are stated simply, clearly and concisely. These statements are supported by empirical evidence, scientific certainty and enjoy wide consensus across influential institutions. The fact that scientists agree that AGW is happening is the safest thing one might say about climate change.

Yet, if public discourse is rationalized to produce this style and tone of discourse, it becomes difficult to address questions engendering ambiguity, uncertainty and ontological insecurity. It is likely that public discussion will drift to those subjects for which facts, certainty and consensus prevail. If discussion of contested topics becomes unavoidable, the norms and expectations of consensus messaging set an impossibly high standard-it is a remarkable accomplishment to find an anthropogenic signal in the global temperature record, but could the same certainty be attained on the relationship of climate change, migration and forced dislocation? As these relationships now mediate our relationship to dangerous climate change, does the desire for clear, certain and scientifically warranted statements encourage researchers to connect climate change and migration using the measures and methodologies having the widest recognition and authority? Has the knowledge and experience of those affected shaped these discussions in an appreciable way?

\section{Symbols of Consensus}

The most pressing difficulty with consensus messaging is the endlessly reiterated symbols of the primary message itself. When climate change danger is addressed primarily through the dominant symbols of consensus messaging, public discourse internalizes the terminology of AGW and conceptualizes danger using the metrics and methodologies that are most familiar, trusted and scientifically authoritative. When this happens, we find an obsessive reliance of the measures of atmospheric carbon dioxide $(\mathrm{CO} 2)$ concentration and global mean surface temperature (GMT) that were compiled, reviewed, re-reviewed and endlessly re-examined to appease skeptics in proving the detection of AGW (Russill, 2016; Pearce et al., 2018). These are among the most studied and certain measurements in all of geophysics; arguably, their accuracy, reliability and authority result from incorporating the challenges of skeptics into the science and policy of climate change. In this respect, the legacy of consensus messaging is not Obama's tweet but the repurposing of GMT to determine the threshold for danger in building political consensus for the Paris Accord.

There are many problems in using GMT to determine a global threshold for danger. It is uses a single metric to organize discussion of a wide array of dangers, many of which can 
have tenuous relationships to GMT. It is a global average and shifts incrementally, a fact representing the approach to danger in a steady and incremental way. It encourages attention to the atmosphere at the expense of the ocean: solar radiation management addresses the increase in GMT in a direct way, but not ocean acidification. And so on.

The point here is not to elevate these debates. It is to recognize how the symbols of consensus messaging circumscribe discussions and regulation of danger. The threats of climate change are articulated in terms of the most familiar and certain elements of AGW, and this distorts communication by foreclosing the symbolism and experiences that could expand the sorts of dangers that are legitimately discussed. It is time to shift the constitutive concerns of climate change communication from responding to skeptics to what is owed those affected most directly and significantly by danger.

\section{CONCLUSION}

Is it possible to see contemporary disputes in climate change communication as symptomatic of the broader discomfort that liberal democracies have in facing the dangers of climate change? The primary problem is not public ignorance of science-whether due to the complexity of the situation or the nefarious tactics of skeptics-but an unwillingness to accept the agonism involved in prioritizing the perspectives of those most affected. The history, experience and knowledge of those migrating or dislocated due to climate change rarely figure into our theories of communication and public life, yet an interest in climate change danger can no longer preclude their involvement. It is not that migrants and refugees are unknown to climate change communication; it is that their usual representation has yet to unsettle the privileging of citizen opinion and participation in public life.

If we recognize that climate change danger will be mediated by questions of migration, dislocation and refuge, and if climate change communication abandons the legacy of consensus messaging to involve those affected by danger, how might our work unfold differently?

There are three implications to letting migration reshape the expectations, styles, and symbolism of climate change communication to accommodate discussions of danger: first, this subject matter infuses public communication with more grounded and complex senses of political urgency than currently emanate from scientists in consensus; second, these concerns make clear that social inequality and political marginalization shape vulnerabilities to climate change danger; and finally, it becomes evident that our usual conceptions of public communication are abstractions from the commitments and ideals animating the political systems of liberal democratic states (and that such communication is relevant primarily to those enjoying the privileges and security afforded by those states). In short, an expanded sense of climate change danger requires moving forcefully beyond the "post-political" approaches of consensus messaging (Carvalho and Peterson, 2012; Machin, 2013; Maeseele, 2015; Carvalho et al., 2017).

One way to explore these implications is to shift the sites we prioritize in generating theories and strategies of climate change communication. Instead of "publics" composed of citizens whose opinions we value as a predictor of political support for policy, or whose participation we desire in shaping alternative futures, we might prioritize "publics" composed by climate change danger; for example, if our point of departure is public health instead of public opinion, then citizen beliefs are deemphasized and considered alongside those cultural and institutional practices mediating the most intense effects of dangerous climate change. This conception of public life is less an effect of modern political theory than constituted from the diverse practices that shape the many sites and scales of migratory and refugee movement (camps, border crossings, immigration and labor laws, primary care clinics, public health funding mechanisms, etc.). In these contexts, migration and dislocation are not framed as effects of extreme weather, natural disaster or national security crisis, but as mediating the challenges of climate change adaptation and forcing climate change communication to ask what it contributes (or owes) in these contexts. It is an approach to communication theory grounded less by political theory than by "dirt research," an orientation developed by Harold Innis for integrating environmental, political and communicative concerns (see Young, 2017).

To conclude, we should address questions of dangerous climate change, ensure the agonism generated by inequality and marginality is understood as mediating vulnerability to danger, and reconsider the communicative practices, contexts and concerns that take priority in our work. In taking up migration and refugee concerns within this context, a wider range of experiences circulate and the requirements of more diverse organizations come into the political foreground. These experiences might dislodge citizens as the privileged subject of political discourse, and refuse the usual mechanisms by which migrants and refugees become legible in liberal democracies. In this respect, migration and refugee concerns are not frames gaining new salience to shape citizen opinion; they are the exclusions through which climate change communication constitutes the politics of danger around the privilege and protection of citizens. We should unsettle this assumption and prioritize what is owed to those most affected by climate change danger-wherever and whenever that might be-or ask why we have said so little on this question. Climate change isn't safe; its communication shouldn't be either.

\section{AUTHOR CONTRIBUTIONS}

The author confirms being the sole contributor of this work and approved it for publication. 


\section{REFERENCES}

Berglez, P., and Olausson, U. (2014). The post-political condition of climate change: an ideology approach. Capit. Nat. Soc. 25, 54-71. doi: 10.1080/10455752.2013.845588

Carvalho, A., and Peterson, T. R. (eds) (2012). Climate Change Politics: Communication and Public Engagement. Amherst, NY: Cambria Press.

Carvalho, A., van Wessel, M., and Maeseele, P. (2017). Communication practices and political engagement with climate change: a research agenda. Environ. Commun. 11, 122-135. doi: 10.1080/17524032.2016.1241815

Cook, J., and Lewandowsky, S. (2016). Rational irrationality: modeling climate change belief polarization using Bayesian networks. Top Cogn. Sci. 8, 160-179. doi: $10.1111 /$ tops.12186

Cook, J. D., Nuccitelli, D., Skuce, A., Jacobs, P., Painting, R., Honeycutt, R., et al. (2014). Reply to 'quantifying the consensus on anthropogenic global warming in the scientific literature: a re-analysis'. Energy Policy 73, 706-708. doi: 10.1016/j.enpol.2014.06.002

Cook, J. D., Nuccitelli, S. A., Green, M., Richardson, B., Winkler, R., Painting, R., et al. (2013). Quantifying the consensus on anthropogenic global warming in the scientific literature. Environ. Res. Lett. 8:024024. doi: 10.1088/1748-9326/8/2/024024

Cook, J. D., Oreskes, N., Doran, P. T., Anderegg, W. R. L., Anderegg, B., Maibach, E. W., et al. (2016). Consensus on consensus: a synthesis of consensus estimates on human-caused global warming. Environ. Res. Lett. 11, 1-7. doi: $10.1088 / 1748-9326 / 11 / 4 / 048002$

Deryugina, T., and Shurchkov, O. (2016). The effect of information provision on public consensus about climate change. PLOS ONE 11:e0151469. doi: 10.1371/journal.pone.0151469

Ding, D., Maibach, E. M., Zhao, X., Roser-Renouf, C., and Leiserowitz, A. (2011). Support for climate policy and societal action are linked to perceptions about scientific agreement. Nat. Clim. Change 1, 462-465. doi: 10.1038/nclimate1295

Heymann, M., Gramelsberger, G., and Mahony, M. (2017). Key Characteristics of Cultures of Prediction. Cultures of Predication in Atmospheric and Climate Science: Epistemic and Cultural Shifts in Computer-Based Modeling and Simulation. New York, NY: Routledge.

Howe, J. P. (2014). Behind the Curve: Science and the Politics of Global Warming. Seattle, DC: University of Washington Press.

Hulme, M. (2009). Why We Disagree About Climate Change. Nottingham, UK: Cambridge University Press.

Hulme, M. (2013). Exploring Climate Change Through Science and Society. New York, NY: Routledge.

Hulme, M. (2015). 'Scientists Speaking With One voice': Panacea or Pathology? Lecture delivered at University of Nottingham, 24 June.

Kahan, D. (2015). Climate-science communication and the measurement problem. Political Psychol. 36, 1-43. doi: 10.1111/pops.12244

Kahan, D., Jenkins-Smith, H., and Braman, D. (2011). Cultural cognition of scientific consensus. J Risk Res. 4, 147-174. doi: 10.1080/13669877.2010.511246

Machin, A. (2013). Negotiating Climate Change: Radical Democracy and the Illusion of Consensus. London: Zed Books.

Maeseele, P. (2013). "On media and science in late modern societies," in Communication Yearbook, ed E. Cohen (New York, NY: Routledge), 154-181.

Maeseele, P. (2015). "Beyond the post-political zeitgeist," in The Routledge Handbook of Environment and Communication, eds A. Hansen and R. Cox (New York, NY: Routledge), 429-443.

Maibach, E., Myers, T., and Leiserowitz, A. (2014). Climate scientists need to set the record straight: there is a scientific consensus that human-caused climate change is happening. Earth's Fut. 2, 295-298. doi: 10.1002/2013EF 000226

McCright, A. M., Dunlap, R. E., and Xiao, C. (2013). Perceived scientific agreement and support for government action on climate change in the USA. Clim. Change 119, 511-518. doi: 10.1007/s10584-013-0704-9

Obama, B. (2013). Twitter [tweet, shared link]. 2013 May 16, 1:48 p.m. [Accessed August 23, 2018]. Available online at: https://twitter.com/barackobama/status/ 335089477296988160

Oreskes, N. (2004). Beyond the ivory tower: the scientific consensus on climate change. Science 306:1686. doi: 10.1126/science.1103618

Pearce, W., Grundmann, R., Hulme, M., Raman, S., Kershaw, E. H., and Tsouvalis, J. (2017). Beyond counting climate consensus. Environ. Commun. 723-730.

Pearce, W., Mahony, M., and Raman, S. (2018). Science advice for global challenges: learning from trade-offs in the IPCC. Environ. Sci. Policy 80, 125-131. doi: 10.1016/j.envsci.2017.11.017

Powell, J. L. (2015). Climate scientists virtually unanimous: anthropogenic global warming is true. Bull. Sci. Technol. Sci. 35, 121-124. doi: $10.1177 / 0270467616634958$

Powell, J. L. (2016). The consensus on anthropogenic global warming matters. Bull. Sci. Technol. Soc. 36, 157-163. doi: 10.1177/0270467617707079

Russill, C. (2016). "The climate of communication: from detection to danger," in Reframing Climate Change: Constructing Ecological Politics, eds O'Lear and S. Dalby (New York, NY: Routledge), 30-51.

Sarewitz, D. (2011). Does climate change knowledge really matter? WIREs Clim Change 2, 475-481. doi: 10.1002/wcc.126

Skuce, A. G., Cook, J., Richardson, M., Winkler, B., Rice, K., Green, S. A., et al. (2016). Does it matter if the consensus on anthropogenic global warming is $97 \%$ or 99.99\%? Bull. Sci. Technol. Soc. 36, 150-156. doi: 10.1177/0270467617702781

Tol, R. (2014). Quantifying the consensus on anthropogenic global warming in the literature: a reanalysis. Energy Policy 73:709. doi: 10.1016/j.enpol.2014.06.003

Tol, R. (2016). Comment on 'Quantifying the consensus on anthropogenic global warming in the scientific literature. Environ. Res Lett. 11, 1-6. doi: 10.1088/1748-9326/11/4/048001

van der Linden, S. L., Leiserowitz, A. A., Feinberg, G. D., and Maibach, E. W. (2014). How to communicate the scientific consensus on climate change: plain facts, pie charts or metaphors? Clim. Change 126, 255-262. doi: 10.1007/s10584-014-1190-4

van der Linden, S. L., Leiserowitz, A. A., Feinberg, G. D., and Maibach, E. W. (2015). The scientific consensus on climate change as a gateway belief: experimental evidence. PLoS ONE 10:e0118489. doi: 10.1371/journal.pone.0118489

Weart, S. R. (2003). The Discovery of Global Warming. Cambridge, MA: Harvard University Press.

Young, L. C. (2017). Innis's infrastructure: dirt, beavers, and documents in material media theory. Cult. Politics 13, 227-249. doi: 10.1215/17432197-4129161

Conflict of Interest Statement: The author declares that the research was conducted in the absence of any commercial or financial relationships that could be construed as a potential conflict of interest.

Copyright (๑) 2018 Russill. This is an open-access article distributed under the terms of the Creative Commons Attribution License (CC BY). The use, distribution or reproduction in other forums is permitted, provided the original author(s) and the copyright owner(s) are credited and that the original publication in this journal is cited, in accordance with accepted academic practice. No use, distribution or reproduction is permitted which does not comply with these terms. 